\title{
Extracellular Signals of a Human Epithelial Colorectal Adenocarcinoma (Caco-2) Cell Line Facilitate the Penetration of Pseudomonas aeruginosa PAO1 Strain through the Mucin Layer
}

\author{
Naoki Hayashi ${ }^{\star}$, Atsushi Yokotani, Masami Yamamoto, Mariko Kososhi, Mayu Morita, \\ Chiaki Fukunishi, Nagisa Nishizawa and Naomasa Gotoh
}

Department of Microbiology and Infection Control Science, Kyoto Pharmaceutical University, Kyoto, Japan

OPEN ACCESS

Edited by:

Matthew C. Wolfgang, University of North Carolina at Chapel

Hill, United States

Reviewed by:

Sang Sun Yoon,

Yonsei University, South Korea

Hongwei David Yu,

Joan C. Edwards School of Medicine,

Marshall University, United States

*Correspondence: Naoki Hayashi

nhayashi@mb.kyoto-phu.ac.jp

Received: 07 June 2017 Accepted: 06 September 2017 Published: 21 September 2017

Citation:

Hayashi $N$, Yokotani A, Yamamoto $M$, Kososhi M, Morita M, Fukunishi C, Nishizawa N and Gotoh N (2017) Extracellular Signals of a Human Epithelial Colorectal Adenocarcinoma

(Caco-2) Cell Line Facilitate the

Penetration of Pseudomonas aeruginosa PAO1 Strain through the

Mucin Layer

Front. Cell. Infect. Microbiol. 7:415. doi: $10.3389 / f c i m b .2017 .00415$
Pseudomonas aeruginosa can penetrate the layer of mucus formed by host intestinal epithelial cells, often resulting in sepsis in immunocompromised patients. We have previously demonstrated that $P$. aeruginosa can penetrate the mucin layer by flagellar motility and the degradation of the mucin layer. However, it remains unclear how $P$. aeruginosa initially recognizes epithelial cells. Using the human epithelial colorectal adenocarcinoma (Caco-2) cell line, we investigated extracellular signaling that could facilitate the penetration of $P$. aeruginosa through the mucin layer. The supernatant from Caco-2 cell cultures increased penetration of $P$. aeruginosa through an artificial mucin layer. The Caco-2 cell supernatant increased bacterial flagella-dependent swarming motility, but it did not influence $P$. aeruginosa growth or protease activity. Filtering of the Caco-2 cell supernatant indicated that proteins weighing $<10 \mathrm{kDa}$ increased mucin penetration, swarming motility, and, based on a tethered cell assay, induced acceleration of the flagellar filament rotational rate. Furthermore, a capillary assay showed that $<10$ $\mathrm{kDa}$ proteins in the Caco-2 cell supernatant attracted $P$. aeruginosa cells. Finally, we identified that growth-regulated oncogene- $\alpha(\mathrm{GRO}-\alpha)$ secreted by Caco-2 cells was a factor facilitating flagellar filament rotation and swarming motility, although it did not attract the bacteria. We conclude that penetration of the mucin layer by $P$. aeruginosa is facilitated by small proteins $(<10 \mathrm{kDa})$ secreted by Caco-2 cells, both by inducing acceleration of flagellar motility and increasing chemotaxis.

Keywords: Pseudomonas aeruginosa, human colorectal adenocarcinoma cell line, flagellar motility, chemotaxis, translocation, sepsis, mucin, growth regulated oncogene- $\alpha$

\section{INTRODUCTION}

The translocation of pathogens from the gut into the blood requires them to cross at least two barriers: the mucus layer and the tight junctions formed by epithelial cells (Dharmani et al., 2009; Tlaskalova-Hogenova et al., 2011). The mucus layer, which mainly comprises mucin protein, forms the first barrier between gut contents and epithelial cells (Dharmani et al., 2009). 
The epithelial cell layer, reinforced by tight junctions between the cells, forms the second barrier between the host and the environment (Tlaskalova-Hogenova et al., 2011). Moreover, host defenses include complex mechanisms of the innate immune system, such as phagocytosis by macrophages, dendritic cells, and neutrophils. These cells are attracted to the infection site by chemokines and play an important role in removing pathogens (Janeway and Medzhitov, 2002; Gellatly and Hancock, 2013; Sallenave, 2014). The Gram-negative bacterium Pseudomonas aeruginosa is a major opportunistic pathogen that causes severe infections, such as pneumonia and bacteremia, in immunocompromised patients. Both clinical (Ohara and Itoh, 2003; Shimizu et al., 2006; Vincent et al., 2009) and basic (Koh et al., 2010; Markou and Apidianakis, 2014) research suggests that the gastrointestinal tract is one of the reservoirs for $P$. aeruginosa in immunocompromised patients. Better understanding of opportunistic $P$. aeruginosa infections requires clarification of the mechanisms by which it penetrates the protective barriers and escapes the immune system defenses.

We consider the penetration of $P$. aeruginosa through the epithelial tissue to be at least a five-step process involving the following aspects: (i) recognition of epithelial cells, (ii) access to epithelial cells, (iii) adhesion to epithelial cells, (iv) formation of a permeation route, and ( $\mathrm{v}$ ) migration to a basolateral site (Okuda et al., 2010; Hayashi et al., 2013, 2015; Shikata et al., 2016). We have previously demonstrated that $P$. aeruginosa can penetrate the mucin layer by flagellar motility and mucin degradation (Hayashi et al., 2013). We subsequently showed that the type III effector molecule ExoS facilitates $P$. aeruginosa penetration through the epithelial cell layer by impairing the function of tight junctional proteins in their defense against bacterial penetration (Okuda et al., 2010). We recently showed that injection of ExoS into epithelial cells by $P$. aeruginosa is required for type IV pilus retraction (Hayashi et al., 2015; Shikata et al., 2016). However, the initial step of the entire process, that is how $P$. aeruginosa recognizes the epithelial cells in the first place, remains unclear.

$P$. aeruginosa flagellar motility, either swarming or swimming, derives from the rotation of the flagellar filament, which mainly comprises the major flagellum subunit protein FliC (Rashid and Kornberg, 2000; Macnab, 2003). The flagellar motor complex, MotAB and MotCD, provides energy for the rotational torque of the filament, which acts as a propeller (Doyle et al., 2004; Toutain et al., 2005). Deletion of the type IV pilus filament gene (pilA) increased spreading on swarming agar (Murray and Kazmierczak, 2008). Chemotaxis is required for flagellar filament rotation, and this motility allows $P$. aeruginosa to seek high levels of nutrients or to escape toxic compounds (Kato et al., 2008; Sampedro et al., 2015). Moreover, most $P$. aeruginosa isolates are motile, but even non-motile isolates can cause lethal endogenous bacteremia in leukopenic mice (Hatano et al., 1996). In the present study, we used a human epithelial colorectal adenocarcinoma (Caco2) cell line to examine how $P$. aeruginosa recognizes mucosal epithelial cells so that it can then penetrate through the mucin layer.

\section{MATERIALS AND METHODS}

\section{Bacterial Strains, Plasmids, and Growth Conditions}

Our laboratory stock strains of $P$. aeruginosa are wild-type PAO1 (Stover et al., 2000), $\triangle$ fliC (Hayashi et al., 2013), $\Delta$ motABCD (Hayashi et al., 2013), and $\Delta$ pilA (Okuda et al., 2013). A green fluorescent protein (GFP) plasmid was constructed by subcloning a 700-bp EcoRI digested fragment containing the $g f p$ gene derived from pGreen (Miller and Lindow, 1997) into the EcoRI site of pME6012 (Heeb et al., 2000). The resulting plasmid (pGFP) was then transfected into the wild-type PAO1 strain. $P$. aeruginosa, which were grown in Luria-Bertani (LB) broth (Nippon Becton Dickinson Company, Tokyo, Japan) or on LB agar plates (Nippon Becton Dickinson Company) at $37^{\circ} \mathrm{C}$ and supplemented with $50 \mu \mathrm{g} / \mathrm{ml}$ tetracycline (Sigma-Aldrich Co., St. Louis, MO, USA) when necessary.

\section{Preparation of Caco-2 Cell Supernatants}

The Caco-2 cells were routinely grown at $37^{\circ} \mathrm{C}$ in a $95 \%$ air$5 \% \mathrm{CO}_{2}$ atmosphere in Dulbecco's modified Eagle medium (DMEM) with high glucose (Sigma-Aldrich Co) and 10\% heatinactivated $\left(56^{\circ} \mathrm{C}, 30 \mathrm{~min}\right)$ fetal bovine serum (FBS; Gibco, Grand Island, NY, USA). The cells were harvested using trypsinEDTA (Gibco) and suspended with DMEM-10\% FBS to stop the reaction with trypsin-EDTA. The cells were collected from the cell suspension by a centrifugation, and were washed with Dulbecco's phosphate buffered saline (DPBS; Gibco). Then, the cells were resuspended with DMEM without any serum, and the mixture was centrifuged twice. Finally, the cellular suspension in DMEM was adjusted to $1.0 \times 10^{5}$ cells per 75 $\mathrm{cm}^{2}$ of the tissue culture flask (Thermo Scientific, Waltham, MA, USA). The culture medium was replaced every 5 days, and the supernatant collected after 5, 10, and 15 days of culture was used in subsequent experiments. The collected supernatant was filtered with Centriprep YM-3 or YM-10 (3 or $10 \mathrm{kDa}$; Millipore, Bedford, MA, USA). For some experiments, $500 \mu \mathrm{l}$ of the various Caco-2 cell supernatant samples were treated with $25 \mu l$ of Immobilized TPCK Trypsin (Thermo Fisher) for $1 \mathrm{~h}$ at $37^{\circ} \mathrm{C}$; the Immobilized TPCK Trypsin was then removed by centrifugation $(10,000 \times \mathrm{g}$ for $10 \mathrm{~min})$.

\section{Mucin Penetration Assay}

We undertook an artificial mucin penetration assay as described previously (Hayashi et al., 2013). Mucin chambers were prepared by adding $50 \mu \mathrm{l}$ of $3 \%$ bovine submaxillary mucin (SigmaAldrich Co) in DMEM to Transwell filter units containing 0.143 $\mathrm{cm}^{2}$ porous filter membranes $(3.0-\mu \mathrm{m}$ pores; Corning, New York, NY, USA). Next, $25 \mu \mathrm{l}$ of $P$. aeruginosa culture (5.0 $\times 10^{4}$ colony-forming units; CFU) was pipetted onto the top of the mucin layers and $235 \mu \mathrm{l}$ aliquots of the Caco- 2 cell supernatant samples or DMEM as a control were placed in the bottom chambers of Transwell. After incubation for 3, 4, or $5 \mathrm{~h}$, the bacteria were collected from the bottom chamber. Appropriate dilutions were spread on LB agar plates, incubated at $37^{\circ} \mathrm{C}$ overnight, and CFUs were counted to quantify the bacteria. 


\section{Swarming Assay}

The bacterial motility swarming assay was modified from a previously reported method (Rashid and Kornberg, 2000) with the various Caco-2 supernatant samples (final: diluted to $50 \%$ with water) containing $0.5 \%$ (w/vol) bacto agar (Nippon Becton Dickinson Company) being used to assess swarming motility. A culture of the $P$. aeruginosa PAO1 strain was spotted on the center of the agar plate. The plate was incubated at $37^{\circ} \mathrm{C}$ in $5 \% \mathrm{CO}_{2}$ for $15 \mathrm{~h}$. After incubation, the radial distance $(\mathrm{mm})$ from the center of the agar was measured.

\section{Measurement of Bacterial Growth}

The $P$. aeruginosa PAO1 strain was incubated in the indicated samples at $37^{\circ} \mathrm{C}$ in $5 \% \mathrm{CO}_{2}$. The optical density $(600 \mathrm{~nm})$ was measured at the indicated times. The optical density under each condition was analyzed.

\section{Measurement of Protease Activity}

The assay was modified from a previously reported method (Kessler et al., 1982). Briefly, the $P$. aeruginosa PAO1 strain was incubated in the indicated samples at $37^{\circ} \mathrm{C}$ in $5 \% \mathrm{CO}_{2}$ for $5 \mathrm{~h}$. After incubation, $50 \mu \mathrm{l}$ of the culture supernatant was added to $1.0 \mathrm{ml}$ of $0.3 \%$ azocasein (Sigma-Aldrich Co) in a solution containing $5 \mathrm{mM}$ Tris-HCI and $0.5 \mathrm{mM} \mathrm{CaCl}_{2}(\mathrm{pH}$ 7.5). The reaction mixture was incubated at $37^{\circ} \mathrm{C}$ for $30 \mathrm{~min}$. Undigested substrate was precipitated with $3.3 \%$ trichloroacetic acid and removed by centrifugation $(10,000 \times \mathrm{g}$ for $10 \mathrm{~min})$. The absorbance $(440 \mathrm{~nm})$ of the supernatant was measured. The absorbance under each condition was analyzed.

\section{Tethered Cell Assay}

The assay was modified from a previously reported method (Qian et al., 2013). A glass slide was coated with Protein A (Biovision, Mountain View, CA, USA) for $30 \mathrm{~min}$ and washed with Dulbecco's phosphate buffered saline (DPBS; Gibco) to remove non-adherent Protein A. Thereafter, the slide was coated with anti-FliC flagellar antibody (Hayashi et al., 2013) for 30 min and washed with DPBS. The P. aeruginosa wild-type PAO1 strain was loaded onto the coated slide and non-tethered cells were rinsed off with DPBS. A cover slip was placed on the slide and the bacterial cells were visualized and recorded as a movie using a microscope with a $100 \times$ objective for recording videos of tethered bacteria (EVOS; Thermo Fisher). Rotational speed was calculated from the number of rotations in $60 \mathrm{~s}$.

\section{Chemotaxis Assay}

We undertook a capillary assay as described previously (Liu and Parales, 2008). A culture of the $P$. aeruginosa PAO1 strain with pGreen, expressing GFP, was centrifuged $(1,800 \times \mathrm{g}$ for $5 \mathrm{~min})$ and washed once with chemotaxis buffer $(10 \mathrm{mM}$ potassium phosphate buffer [pH 7.0], $0.1 \mathrm{mM}$ disodium EDTA). Onemicroliter capillary tubes were filled with the indicated samples. The accumulation of cells in the capillary tip was observed using a fluorescence microscope with a $4 \times$ objective (IX-71; Olympus, Tokyo, Japan). GFP intensities of the photograph were measured using ImageJ software (NIH, Bethesda, MD, USA).

\section{Measurement of Cytokine Concentrations}

The concentration of cytokines in supernatant from Caco-2 cells cultured were determined by using the Multi-Analyte ELISArray Kits (Qiagen, Valencia, CA, USA) and Human GRO alpha (CXCL1) Platinum ELISA (Affymetrix, Santa Clara, CA, USA) by following the manufacturer's instructions.

\section{Chemokines and Antibody}

Recombinant human chemokines, including interleukin-8 (IL8 ); regulated on activation, normal T cell expressed and secreted (RANTES); macrophage inflammatory protein-1 $\alpha$ (MIP1 $\alpha)$; macrophage-derived chemokine (MDC); and growth regulated oncogene- $\alpha(\mathrm{GRO}-\alpha)$ were purchased from PeproTech Inc. (Rocky Hill, NJ, USA). An anti-GRO- $\alpha$ antibody was purchased from Abcam Plc (Cambridge, MA, USA), and normal goat serum was purchased from Wako Pure Chemical Industries Ltd. (Osaka, Japan).

\section{Statistical Analysis}

Statistical analysis, including one-way analysis of variance (ANOVA), Tukey's test, and Student's $t$-test, was performed with EZR (Kanda, 2013), a graphical user interface for R. More precisely, it is a modified version of the $\mathrm{R}$ commander designed to add statistical functions frequently used in biostatistics.

\section{RESULTS}

\section{Caco-2 Cell Culture Supernatant Facilitates the Penetration of $P$. aeruginosa through the Mucin Layer}

To clarify the mechanism by which $P$. aeruginosa recognizes extracellular signals of the intestinal epithelial cells using the human intestinal epithelial Caco-2 cell line (Okuda et al., 2010; Hayashi et al., 2013, 2015; Shikata et al., 2016), we examined the effects of Caco-2 cell culture supernatants on mucin penetration by the $P$. aeruginosa PAO1 strain. After $3 \mathrm{~h}$ of incubation, the bacterial numbers in the bottom chamber of wells with 10 - and 15-day Caco-2 cell supernatant were 1.6 and 2.5 times higher than those with DMEM, respectively (Figure 1A; $P<0.05$ ). The effect of the 5-day supernatant on penetration did not differ significantly from that of DMEM (Figure 1A; $P>0.10$ ). After 3, 4 , and $5 \mathrm{~h}$ of incubation, the numbers of bacteria in the bottom chambers filled with 15-day supernatant were 2.5, 2.2, and 2.3 times greater than those cultured with DMEM, respectively (Figure 1B; $P<0.05$ ).

\section{Characteristic of Caco-2 Cell Signaling Facilitating the Penetration of $P$. aeruginosa through the Mucin Layer}

To characterize Caco-2 cell signals that facilitated the penetration of $P$. aeruginosa through the mucin layer, we performed an artificial mucin layer penetration assay using filtered and/or trypsin-treated 15-day Caco-2 cell supernatants. The bacterial numbers in bottom chambers of Transwell filled with unfiltered and $<10 \mathrm{kDa}$ Caco-2 cell supernatant fractions were 2.5 and 2.7 times higher than those with DMEM of the same fraction size 

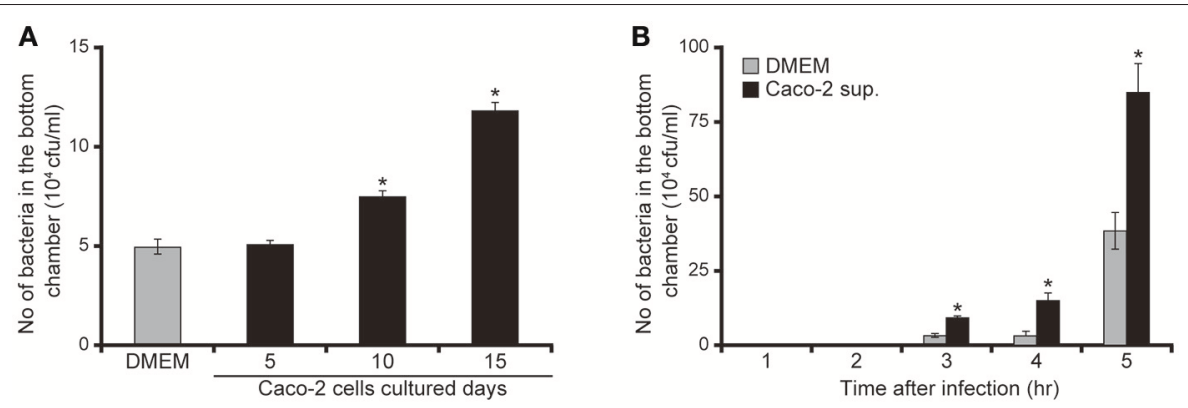

FIGURE 1 | Caco-2 cell supernatant facilitates penetration of the $P$. aeruginosa PAO1 strain through the mucin layer. (A) The bottom chambers of Transwell cells were filled with Caco-2 cell supernatant (collected after 5, 10, and 15 days of culture) or with Dulbecco's modified Eagle medium (DMEM; control). The P. aeruginosa PAO1 strain was added to the top chamber. After $3 \mathrm{~h}$, the numbers of bacteria in the bottom chambers were counted. Error bars indicate standard error $(n=3)$. ${ }^{*} P<0.05$ compared with DMEM. (B) Caco-2 cell supernatant after 15 days of culture (Caco-2 sup.) or DMEM (control) filled the bottom chamber of Transwell cells. After the $P$. aeruginosa PAO1 strain was added, the numbers of bacteria in the bottom chambers were counted at 3,4 , and $5 \mathrm{~h}$. Error bars indicate standard error $(n=3)$. ${ }^{*} P<0.05$ compared with DMEM at the same incubation time.

(Figure 2; $P<0.05$ ). The number of bacteria penetrating the mucin layer did not differ significantly between those treated with $<3 \mathrm{kDa}$ Caco-2 cell supernatant and the DMEM control (Figure 2; $P>0.10$ ). Moreover, trypsin treatment of unfiltered and the $<10 \mathrm{kDa}$ Caco- 2 cell supernatant fraction was associated with less $P$. aeruginosa penetration through the mucin layer (Figure 2).

\section{Caco-2 Cell Supernatant Facilitates Swarming Motility of $P$. aeruginosa}

No significant difference was observed in bacterial growth and protease activity between bacteria incubated with Caco- 2 cell supernatants or the DMEM control (Figures 3A,B; $P>0.10$ ). Both unfiltered Caco-2 cell supernatant and the filtered $<10$ $\mathrm{kDa}$ fraction significantly increased the swarming motility of the $P$. aeruginosa PAO1 strain by 2.7 times (Figures 3C,D; $P<0.05$ ). Trypsin treatment of the supernatant diminished the increase in motility on the swarming agar by the Caco- 2 cell supernatants (Figures 3C,D). P. aeruginosa has two major structures that contribute to motility: the flagella and type IV pili. Deletion of a type IV pilus filament gene (pilA) increased the motility on the swarming agar (Murray and Kazmierczak, 2008). No significant difference was observed in terms of the numbers of either the wild-type PAO1 strain or a mutant with type IV pilus filament deletion (the $\triangle p i l A$ strain) penetrating the mucin layer when treated with either DMEM or Caco-2 cell supernatant (Figure 3E; $P>0.10)$. Penetration of mutants with flagellar filament deletion (the $\triangle f l i C$ strain) and motor deletion (the $\triangle \operatorname{mot} A B C D$ strain) through the mucin layer could not be detected at $3 \mathrm{~h}$ after inoculation (Figure 3E).

\section{Caco-2 Cell Supernatant Facilitates Rotation of the $P$. aeruginosa Flagellar Filament}

Having demonstrated that $<10 \mathrm{kDa}$ proteins secreted by Caco2 cells induced the acceleration of flagellar motility in the $P$. aeruginosa $\mathrm{PAO} 1$ strain, thus facilitating penetration of the mucin layer (Figures 1-3), we sought to clarify the mechanism

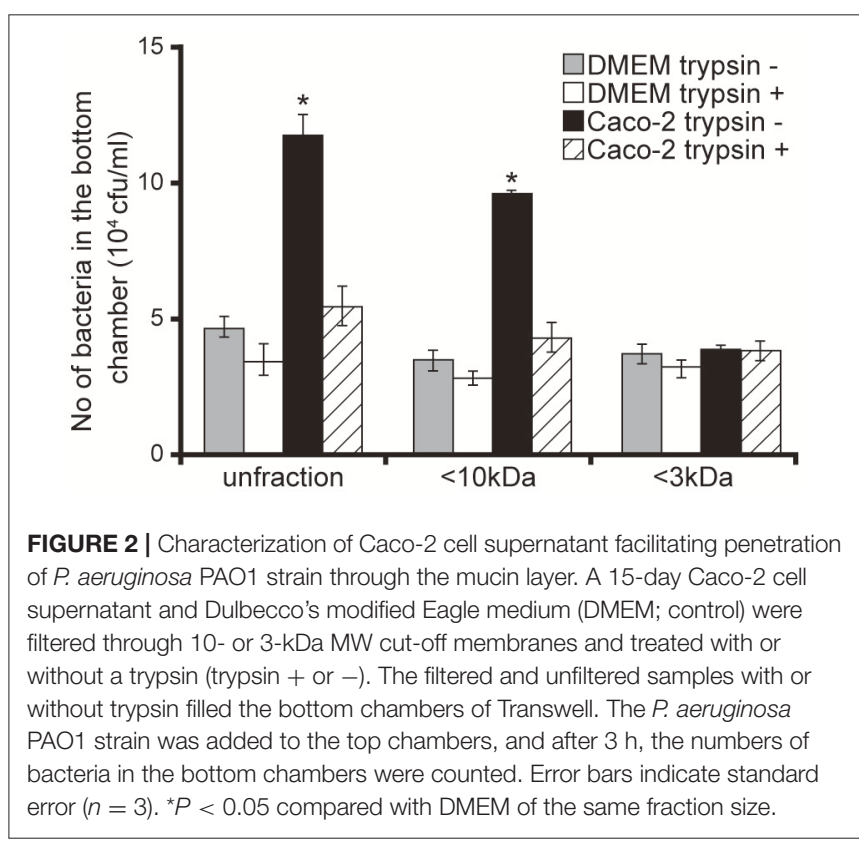

with a tethered cell analysis, measuring the rotational speed of flagellar filaments. The flagellar filament rotational speed of the $P$. aeruginosa PAO1 strain treated with unfiltered and the $<10$ $\mathrm{kDa}$ fraction of the Caco-2 cell supernatant was 1.4 times greater than with DMEM at the same fraction size (Figure 4; $P<0.05$ ). Trypsin treatment of the Caco-2 cell supernatant diminished the increased rotational speed (Figure 4). No significant differences were observed in the number of bacteria adhering to glass slides under all conditions (data not shown).

\section{Caco-2 Cell Supernatant Attracts the $P$. aeruginosa PA01 Strain}

Our capillary assay showed that $10 \mathrm{mM}$ of L-arginine attracted and chloroform repelled $P$. aeruginosa cells (Figures 5A,B; $P<$ 0.05 ). These data were consistent with previous findings (Craven 


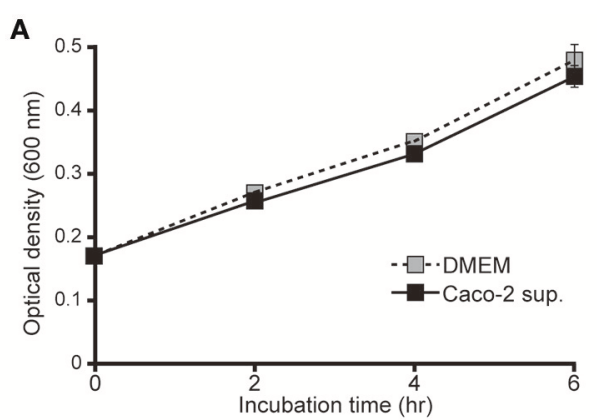

C

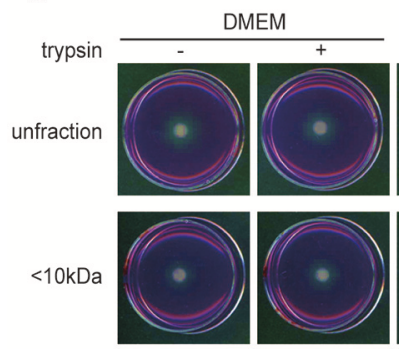

E

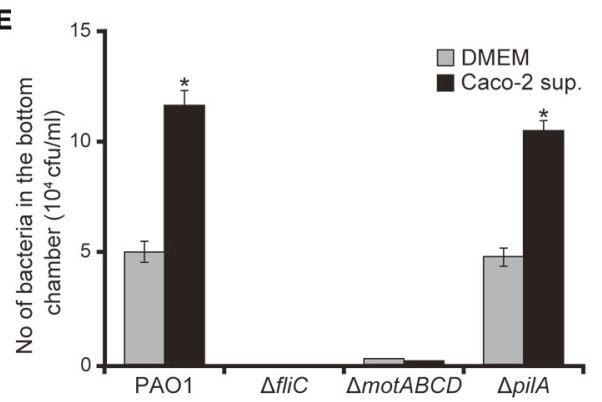

B

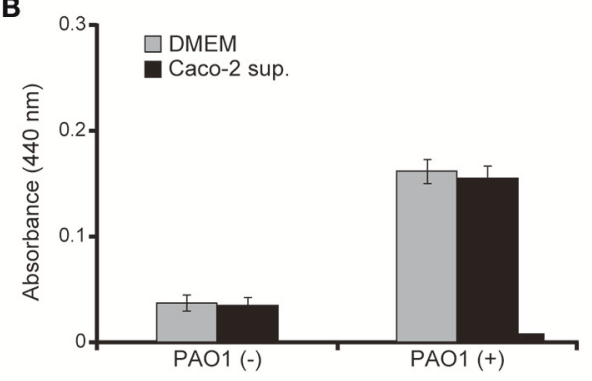

D

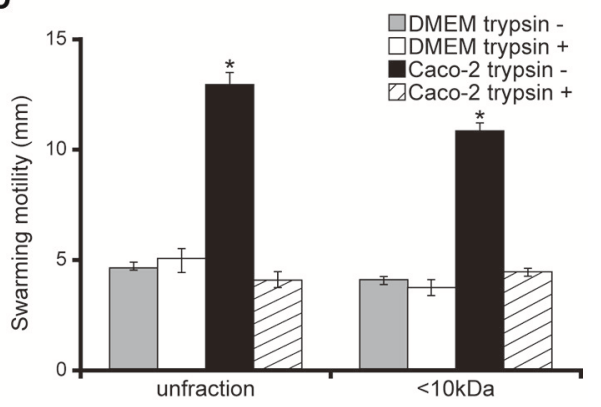

FIGURE 3 | Caco-2 cell supernatant facilitates a swarming motility of the $P$. aeruginosa PAO1 strain. (A) The P. aeruginosa PAO1 strain was incubated in Caco-2 cell supernatant (Caco-2 sup.) or Dulbecco's modified Eagle medium (DMEM; control) at $37^{\circ} \mathrm{C}$ in $5 \% \mathrm{CO}_{2}$. After incubation, the optical density (600 $\mathrm{nm}$ ) of the culture was measured at the indicated times. Error bars indicate standard error $(n=3)$. (B) After incubation of the $P$. aeruginosa PAO1 strain in Caco-2 cell supernatant (Caco-2 sup.) or DMEM (control) at $37^{\circ} \mathrm{C}$ in $5 \% \mathrm{CO}_{2}$ for $5 \mathrm{~h}$, the azocasein degradation activity of the collected culture supernatant was measured. Error bars indicate standard error $(n=3)$. (C) A 15-day Caco-2 cell supernatant (Caco-2 sup.) and DMEM (control) were filtered through 10-kDa MW cut-off membranes. The $<10 \mathrm{kDa}$ fraction and unfiltered samples were treated with or without trypsin (trypsin + or - ). The $P$. aeruginosa PAO1 strain was spotted on swarming agar containing the treated samples. After incubation for $14 \mathrm{~h}$, the swarming agar plates were observed and photographed. (D) The radial distance $(\mathrm{mm})$ from the center of the agar was measured. Error bars indicate standard error $(n=3)$. ${ }^{*} P<0.05$ compared with DMEM at the same size fraction. (E) A 15-day Caco-2 cell supernatant (Caco-2 sup.) or DMEM (control) was filled in the bottom chamber of Transwell. The $P$. aeruginosa PAO1 strain, flagellar mutants ( $\triangle$ fliC and $\triangle m o t A B C D$ ), or a type IV pilus mutant $(\triangle$ pilA $)$ were added to the top chambers, and after $3 \mathrm{~h}$, the numbers of bacteria in the bottom chambers were counted. Error bars indicate standard error $(n=3)$. ${ }^{*} P<0.05$ compared with incubation in DMEM of the same strains.

and Montie, 1985; Shitashiro et al., 2003; Kato et al., 2008). To examine whether the chemoattractant facilitates penetration of $P$. aeruginosa through the mucin layer, we added the chemoattractant or repellent to the bottom chamber of Transwell. After $3 \mathrm{~h}$ of incubation, $10 \mathrm{mM}$ L-arginine significantly increased the number of $P$. aeruginosa in the bottom chamber (Figure $5 \mathrm{C}$; $P<0.05)$. In contrast, chloroform diminished the number of $P$. aeruginosa in the bottom chamber (Figure 5C; $P<$ 0.05). A good correlation was observed between chemotaxis and the penetration of the $P$. aeruginosa PAO1 strain through the mucin layer. Thereafter, to clarify whether the Caco-2 cell supernatant has the ability to attract $P$. aeruginosa, we performed a capillary assay. Both the unfiltered and the $<10 \mathrm{kDa}$ Caco-2 cell supernatant fraction increased GFP intensity in the capillary tubes (Figures 5D,E; $P<0.05$ ). Trypsin treatment of the Caco-2 cell supernatant diminished this increased GFP intensity in the capillary tube (Figures 5D,E; $P<0.05$ ).

\section{Cytokines Secreted by Caco-2 Cells Facilitate the Penetration of the P. aeruginosa PA01 Strain through the Mucin Layer}

Our data suggested that smaller proteins secreted by Caco-2 cells induced the acceleration of flagellar motility required for penetration through the mucin layer (Figures 1-5). We therefore measured various cytokines secreted from Caco-2 cells using enzyme-linked immunosorbent assay (ELISA). As shown in 


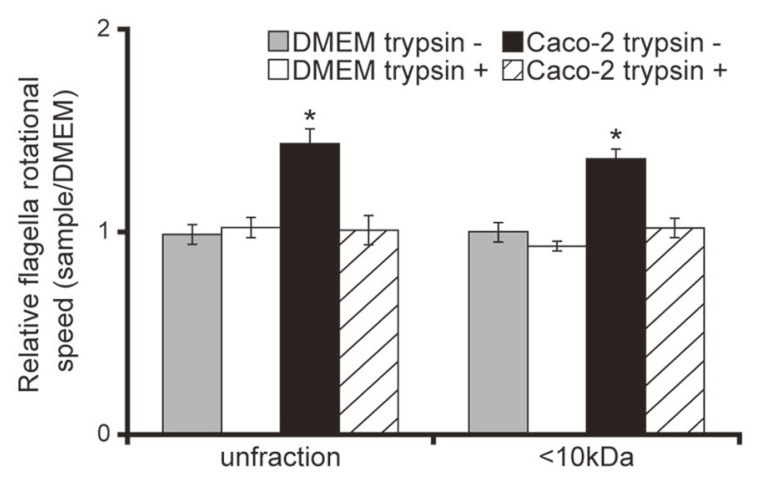

FIGURE 4 | Caco-2 cell supernatant facilitates a flagellar filament rotation of the $P$. aeruginosa PAO1 strain. A 15-day Caco-2 cell supernatant and Dulbecco's modified Eagle medium (DMEM; control) were filtered through $10-\mathrm{kDa}$ MW cut-off membranes. The $<10 \mathrm{kDa}$ fraction or unfiltered samples were treated with or without trypsin (trypsin + or - ). P. aeruginosa PAO1 strain cells treated with the various samples were loaded onto a glass slide precoated with flagellar filament FliC antibodies. Bacterial cells were visualized and recorded as a movie using EVOS microscope, showing videos of tethered bacteria. The rotational speed was calculated from the number of rotations in 60 s. Error bars indicate standard error $(n=10) .{ }^{\star} P<0.05$ compared with DMEM of the same fraction size.
Figure 6A, five cytokines were detected in the 15-day Caco2 cell supernatant (Figure 6A), IL-8 $(112.2 \mathrm{pg} / \mathrm{mL})$, RANTES $(162.9 \mathrm{pg} / \mathrm{mL}), \mathrm{MIP} 1 \alpha,(45.4 \mathrm{pg} / \mathrm{mL}), \mathrm{MDC}(221.3 \mathrm{pg} / \mathrm{mL})$, and GRO- $\alpha(83.4 \mathrm{pg} / \mathrm{mL})$ (Figure 6A). No cytokines were detected in DMEM (data not shown). We subsequently investigated whether the chemokines in the Caco-2 cell supernatant could have facilitated the penetration of $P$. aeruginosa through the artificial mucin layer. We added each of the five chemokines at the concentrations detected in the Caco- 2 cell supernatant to the bottom chambers of Transwell and assessed the penetration of P. aeruginosa. Of the five chemokines, only GRO- $\alpha$ significantly increased the number of bacteria, by 2.0 -fold; the Caco2 cell supernatant increased 2.3-fold (Figure 6B; $P<0.05$ ). Furthermore, addition of anti- GRO- $\alpha$ antibodies into Caco2 cells supernatant significantly decreased penetration through mucin layers (Figure 6C; $P<0.05$ ). The effect of normal goat serum on penetration did not differ significantly from that of Caco-2 cells supernatant (Figure 6C; $P>0.10$ ). GRO$\alpha$ concentrations in the Caco-2 cell supernatant increased with the length of Caco-2 culture (Figure 6D; $P<0.05$ ). A good correlation was observed between the increase of GRO- $\alpha$ concentration and that in mucin penetration by $P$. aeruginosa (Figures 1A, 6D).
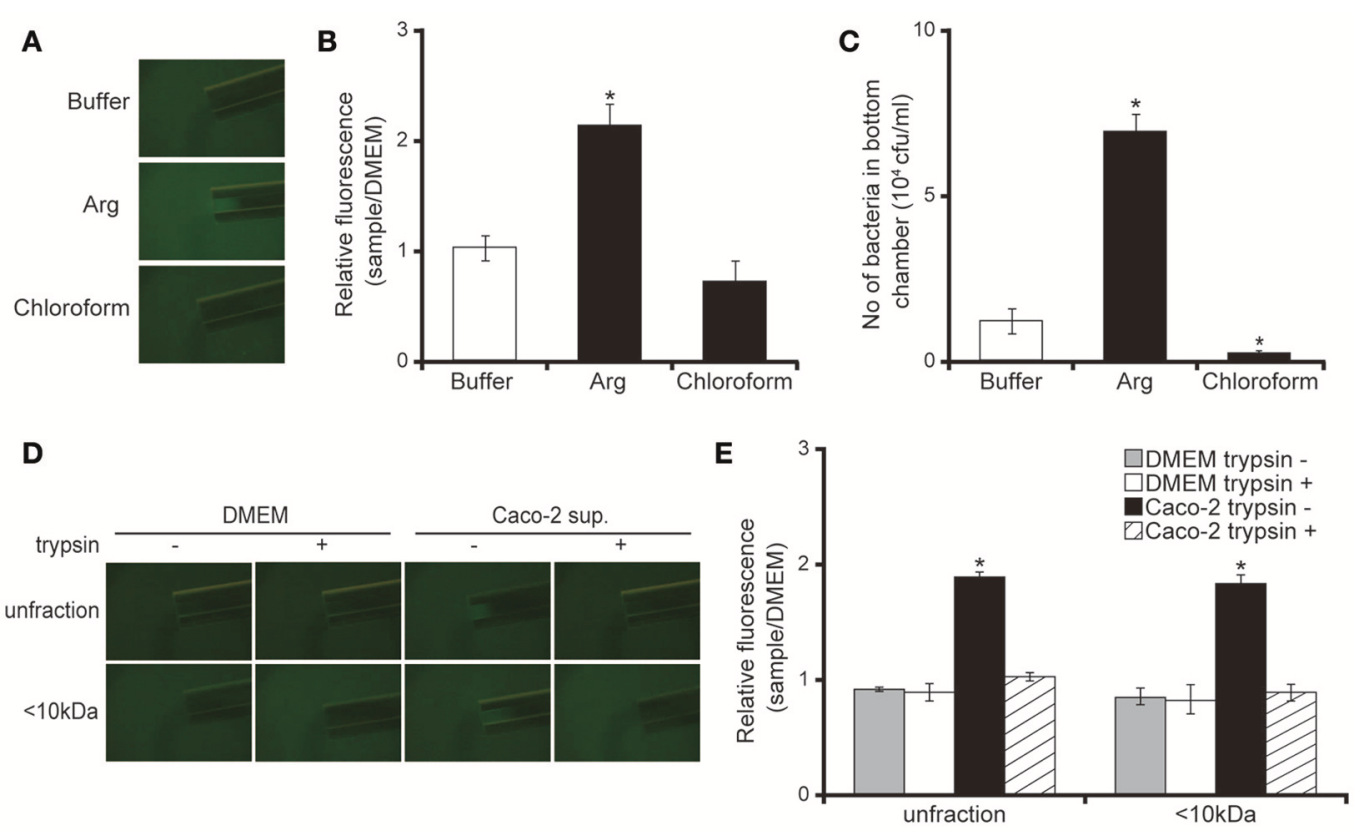

FIGURE 5 | Caco-2 cell supernatant attracts the $P$. aeruginosa PAO1 strain. (A) A glass slide surface was filled with a $P$. aeruginosa PAO1 strain expressing a green fluorescent protein (GFP). Capillary tubes were filled with buffer (control), Arg (attractant), or chloroform (repellent). The accumulation of the $P$. aeruginosa PAO1 strain expressing GFP at the capillary tip was visualized and photographed by microscopy at a magnification of $40 \times$. (B) The graph shows the relative intensity of GFP in the capillary after incubation for 10 min. Error bars indicate standard error $(n=3)$. ${ }^{*} P<0.05$ compared with buffer. (C) A buffer (control), Arg (attractant), or chloroform (repellent) filled the bottom chamber of Transwell. The P. aeruginosa PAO1 strain was added and, after $3 \mathrm{~h}$, the numbers of bacteria in the bottom chambers were counted. Error bars indicate standard error $(n=3)$. ${ }^{*} P<0.05$ compared with buffer. (D) A 15-day Caco-2 cell supernatant and Dulbecco's modified Eagle medium (DMEM; control) were filtered through 10-kDa MW cut-off membranes. The $<10 \mathrm{kDa}$ fraction or unfiltered samples were treated with or without trypsin (trypsin + or -). A cover glass surface was filled with the P. aeruginosa PAO1 strain expressing GFP. Capillary tubes were filled with the treated samples. The accumulation of the GFP-expressing P. aeruginosa PAO1 strain at the capillary tip was visualized and photographed by microscopy at a magnification of $40 x$. (E) The graph shows the relative intensity of GFP in the capillary tube after incubation for $10 \mathrm{~min}$. Error bars indicate standard error $(n=3) .{ }^{*} P<0.05$ compared with DMEM of the same fraction size. 


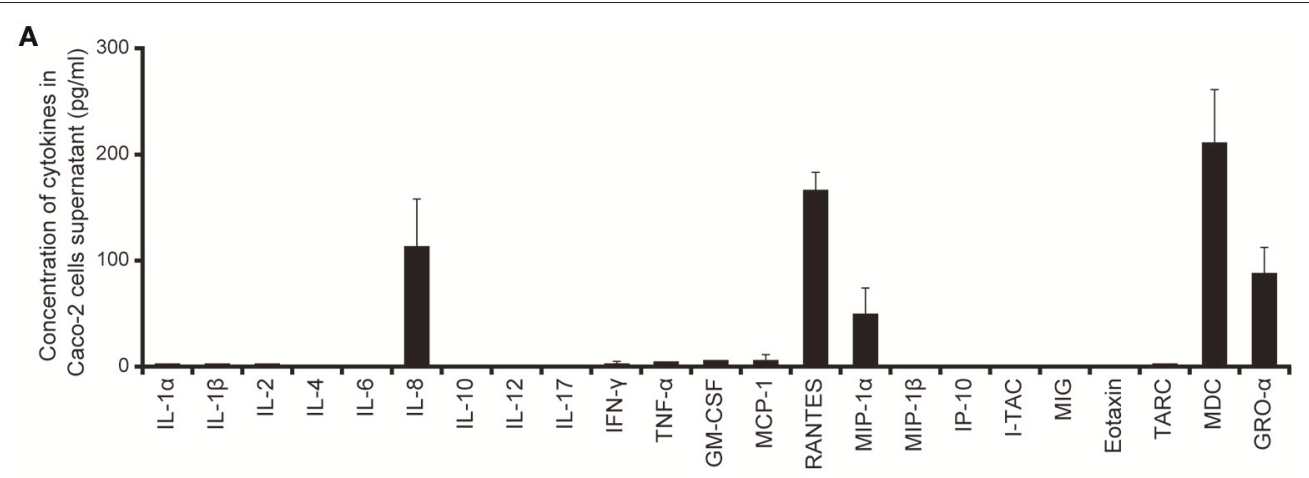

B

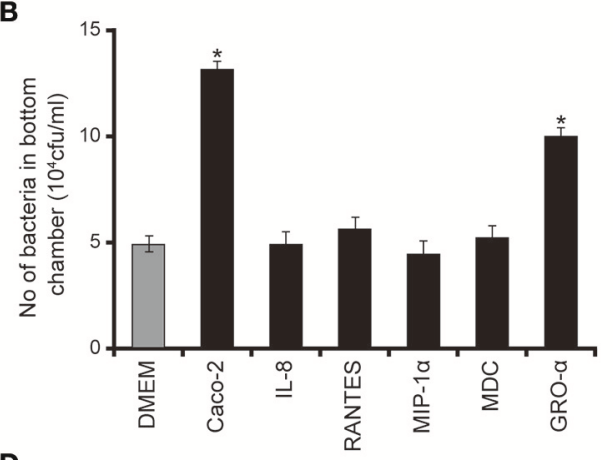

D

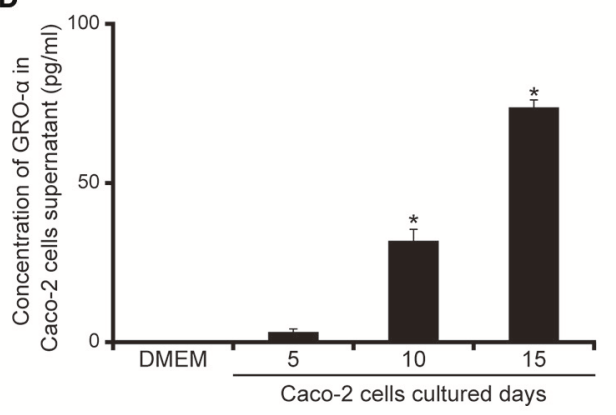

C

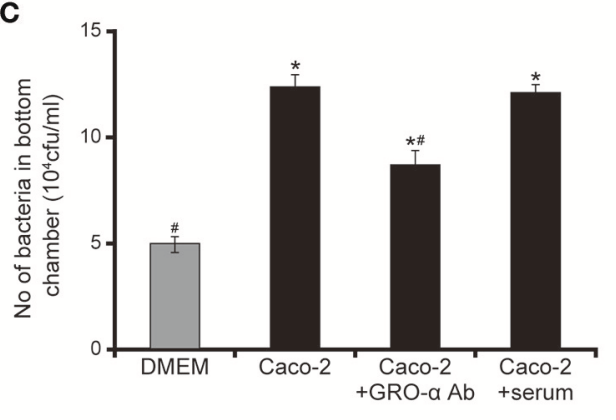

FIGURE 6 | Chemokines secreted by Caco-2 cells facilitate the penetration of the P. aeruginosa PAO1 strain through the mucin layer. (A) Cytokine levels in the 15-day Caco-2 cell supernatant were determined by enzyme-linked immunosorbent assay (ELISA). The graph shows the cytokine concentrations in the supernatant. Error bars indicate standard error $(n=3)$. (B) The cytokines identified by the assay were placed in the bottom chamber of Transwell, as were unfiltered 15 -day Caco-2 cell supernatant and Dulbecco's modified Eagle medium (DMEM; control). The $P$. aeruginosa PAO1 strain was added to the top chambers and, after 3 h, the numbers of bacteria in the bottom chamber were counted. Error bars indicate standard error $(n=3)$. ${ }^{*}<0.05$ compared with DMEM. (C) Caco-2 cells supernatant were pretreated for 30 min with anti-GRO- $\alpha$ antibodies (GRO - $\alpha$ Ab) (1 ml of Caco-2 cells supernatant/100 ng of antibody) or normal goat serum (serum) then inoculated onto the bottom chamber of Transwell. Error bars indicate standard error $(n=3)$. ${ }^{*} P<0.05$ compared with DMEM. $\# P<0.05$ compared with Caco-2 cell supernatant. ${ }^{*} P P<0.05$ compared with DMEM and $P<0.05$ compared with Caco-2 cell supernatant. (D) Growth regulated oncogene- $\alpha$ concentrations in the $5-$, 10-, and 15-day Caco-2 cell supernatants were determined by ELISA. Error bars indicate standard error $(n=3)$. ${ }^{\star} P<0.05$ compared with DMEM.

\section{GRO- $\alpha$ Facilitates Flagellar Motility of the $P$. aeruginosa PA01 Strain}

The addition of GRO- $\alpha(83.4 \mathrm{pg} / \mathrm{mL})$ to swarming agar led to a significant 2.3 -fold increase in the swarming motility of the $P$. aeruginosa PAO1 strain (Figures 7A,B; $P<0.05$ ). Next, we measured the rotational speed of the flagellar filament by a tethered cell analysis. GRO- $\alpha(83.4 \mathrm{pg} / \mathrm{mL})$ led to a significant 1.4-fold increase in the flagellar filament rotation speed of the $P$. aeruginosa PAO1 strain (Figure 8; $P<0.05$ ). No significant difference was observed between GRO- $\alpha(83.4 \mathrm{pg} / \mathrm{mL})$ and DMEM control in the number of bacteria adhering to the glass slides (data not shown). Treatment with GRO- $\alpha$ did not show any difference in increased GFP intensity in capillary tubes (Figures 9A,B; $P>0.10$ ).

\section{DISCUSSION}

In this study, we examined the effects of the supernatant of Caco2 cells cultured in vitro on mucin penetration by the $P$. aeruginosa PAO1 strain to clarify the mechanism by which $P$. aeruginosa recognizes extracellular signals from intestinal epithelial cells to facilitate bacterial translocation. Our results indicated that 
A

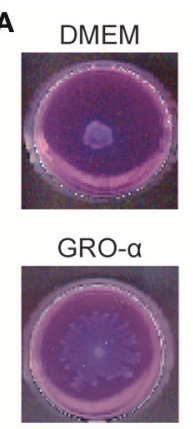

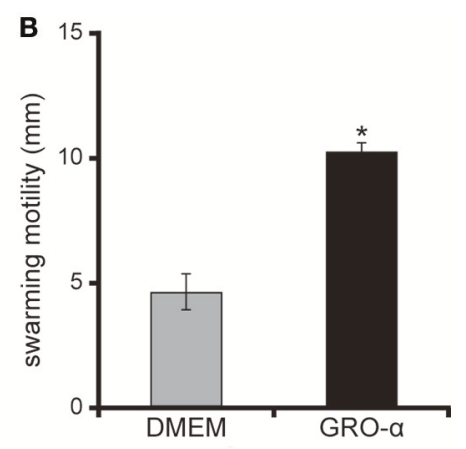

FIGURE 7 | GRO- $\alpha$ facilitates a swarming motility of the $P$. aeruginosa PAO1 strain. (A) The $P$. aeruginosa PAO1 strain was spotted on swarming agar containing growth regulated oncogene $-\alpha$ or Dulbecco's modified Eagle medium (DMEM; control). After incubation for $14 \mathrm{~h}$, the swarming agar plate was observed and photographed. (B) The radial distance $(\mathrm{mm})$ from the center of the agar was measured. Error bars indicate standard error $(n=3)$. ${ }^{\star} P$ $<0.05$ compared with DMEM.

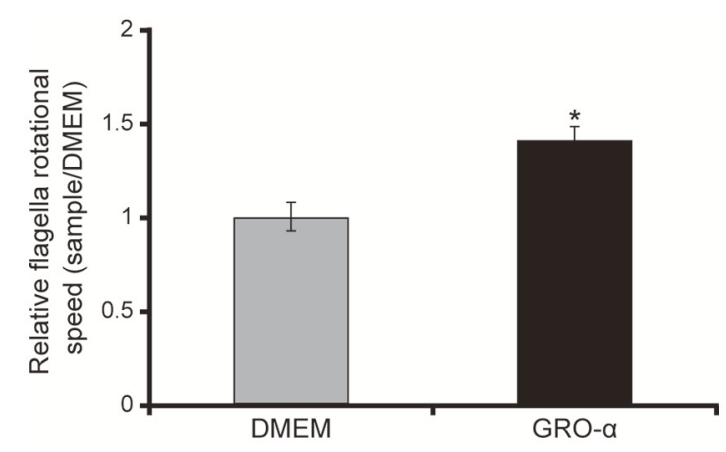

FIGURE 8 | GRO- $\alpha$ facilitates a flagellar filament rotation of the $P$. aeruginosa PAO1 strain. P. aeruginosa PAO1 strain cells were loaded onto a glass slide precoated with flagellar filament FliC antibodies, and growth regulated oncogene- $\alpha$ or Dulbecco's modified Eagle medium (DMEM; control) was added. Bacterial cells were visualized and recorded as a movie using EVOS microscope, showing videos of the tethered bacteria. The rotational speed was calculated from the number of rotations in $60 \mathrm{~s}$. Error bars indicate standard error $(n=10)$. ${ }^{*} P<0.05$ compared with DMEM.

the $<10 \mathrm{kDa}$ proteins secreted by Caco- 2 cells facilitated the penetration of $P$. aeruginosa through the mucin layer. These small proteins enhanced swarming motility but did not influence the growth rate or protease activity of the P. aeruginosa PAO1 strain. We then found that the $<10 \mathrm{kDa}$ proteins secreted by Caco- 2 cells accelerated flagellar filament rotation and attracted the $P$. aeruginosa PAO1 strain into capillary tubes. Among the tested chemokines, GRO- $\alpha$ was found to facilitate mucin layer penetration by $P$. aeruginosa by inducing the acceleration of flagellar motility, although not by increased chemotaxis. We conclude that GRO- $\alpha$ and other unknown $<10 \mathrm{kDa}$ proteins secreted by intestinal epithelial cells facilitate penetration of the mucin layer by $P$. aeruginosa.

We previously demonstrated that $P$. aeruginosa penetrates the mucin layer using flagellar motility and mucin degradation
A
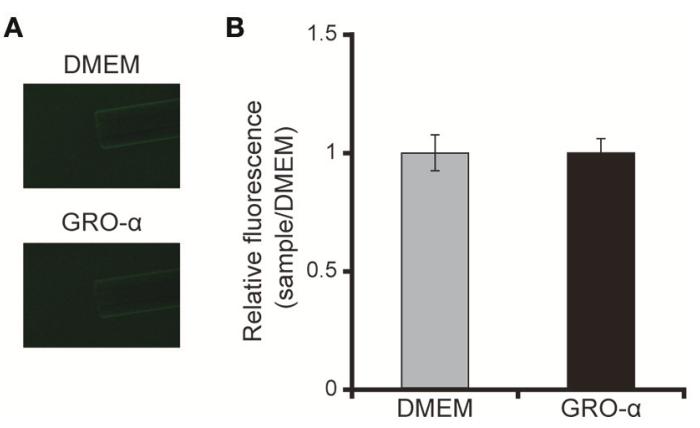

FIGURE 9 | GRO- $\alpha$ does not attract the $P$. aeruginosa PAO1 strain. (A) A glass slide was filled with the GFP-expressing $P$. aeruginosa PAO1 strain. A capillary tube was filled with growth regulated oncogene- $\alpha$ or Dulbecco's modified Eagle medium (DMEM; control). The accumulation of $P$. aeruginosa at the capillary tip was visualized and photographed by microscopy at a magnification of $40 \times$. (B) The graph shows the relative intensity of GFP in the capillary tube after incubation for $10 \mathrm{~min}$. Error bars indicate standard error $(n=3)$.
(Hayashi et al., 2013). The present study showed that Caco-2 cells enhanced flagella-dependent swarming motility but did not influence the growth rate or protease activity of the $P$. aeruginosa PAO1 strain. P. aeruginosa flagellar motility derives from rotation of the flagellar filament, which mainly comprises the major flagellum subunit protein FliC (flagellin) (Rashid and Kornberg, 2000; Macnab, 2003). Some researchers have suggested that three main factors can affect $P$. aeruginosa motility on swarming agar: (i) acceleration of flagellar filament rotation (Rashid and Kornberg, 2000; Macnab, 2003; Doyle et al., 2004; Toutain et al., 2005), (ii) addition of a chemoattractant to the swarming agar (Tremblay et al., 2007), and (iii) deficiency of type IV pili (Murray and Kazmierczak, 2008). As no significant difference was observed in the penetration of the $P$. aeruginosa pilus filament mutant ( $\triangle$ pilA strain) through the mucin layers with or without the Caco-2 cell supernatant, it is suggested that the Caco-2 cell supernatant does not affect the function of the type IV pili in relation to flagellar motility. However, the Caco2 cell supernatant did significantly accelerate flagellar filament rotation, as well as exhibit chemoattraction for the $P$. aeruginosa PAO1 strain. Thus, collectively, our novel findings suggest that Caco-2 cells facilitate $P$. aeruginosa penetration through the mucin layer by at least two mechanisms: acceleration of flagellar filament rotation and chemotaxis.

Our data showed that $<10 \mathrm{kDa}$ proteins secreted by Caco2 cells facilitated the penetration of $P$. aeruginosa through the mucin layer, along with its swarming motility. Moreover, the supernatant filtrate containing these $<10 \mathrm{kDa}$ proteins had the same effect as the unfiltered supernatant on flagellar filament rotation and chemotaxis. The mucin penetration and swarming motility in addition of GRO- $\alpha$ alone in the same concentration in Caco- 2 cell supernatant showed the 75 and $78 \%$ in addition of Caco-2 cell supernatant, respectively. Then, we found that GRO- $\alpha$ alone in the same concentration as that in the Caco-2 cell supernatant had an even greater effect than the supernatant alone on flagellar filament rotational speed. It did not, however, 
attract $P$. aeruginosa cells. Taken together, our data suggest that Caco- 2 cells secrete several factors that affect $P$. aeruginosa. GRO$\alpha$, whether alone or along with other unidentified substances, facilitates flagellar filament rotation. However, there must also be one or more unknown small proteins serving as chemoattractants for $P$. aeruginosa. Further studies are necessary to identify other attractants secreted by Caco- 2 cells.

Our data revealed that $<10 \mathrm{kDa}$ signals secreted by Caco- 2 cells, including GRO- $\alpha$ and other unidentified ones, facilitated the flagellar motility. P. aeruginosa flagellar motility is derived from flagellar filament rotation by flagellar motor complexes, MotAB and MotCD (Rashid and Kornberg, 2000; Macnab, 2003; Doyle et al., 2004; Toutain et al., 2005), but previous reports about a receptor directly regulating the function of the flagellar motor complex in $P$. aeruginosa are absent. On the contrary, receptors regulating the chemotaxis of $P$. aeruginosa have been identified (Kato et al., 2008; Sampedro et al., 2015). Further studies are necessary to identify the receptors in $P$. aeruginosa for the signals secreted by Caco- 2 cells.

Pathogenic bacteria possess virulence factors that facilitate escape from host defense mechanisms. The relation between cytokine production and the establishment of bacterial infection has been examined. Most reports, including investigations of $P$. aeruginosa infections, have focused on the systems by which host cells recognize pathogens or pathogens escape this immune surveillance (Janeway and Medzhitov, 2002; Gellatly and Hancock, 2013; Sallenave, 2014). In contrast, Wu et al. suggested that pathogens may actively respond to a host's immune signals. They showed that interferon-gamma binds to a $P$. aeruginosa outer membrane protein, OprF, resulting in the expression of PA-I lectin-dependent cytotoxicity directed toward epithelial cells (Wu et al., 2005). GRO- $\alpha$ has been shown to attract neutrophils to sites of infection (Bechara et al., 2007), but there have previously been no reports of GRO- $\alpha$ directly affecting $P$. aeruginosa virulence. A novel finding of our study is the role of GRO- $\alpha$ in promoting $P$. aeruginosa virulence by augmenting

\section{REFERENCES}

Bechara, C., Chai, H., Lin, P. H., and Chen, C. (2007). Growth related oncogenealpha (GRO- $\alpha)$ : Roles in atherosclerosis, angiogenesis and other inflammatory conditions. Med. Sci. Monit. 13, RA87-RA90.

Craven, R., and Montie, T. C. (1985). Regulation of Pseudomonas aeruginosa chemotaxis by the nitrogen source. J. Bacteriol. 164, 544-549.

Dharmani, P., Srivastava, V., Kissoon-Singh, V., and Chadee, K. (2009). Role of intestinal mucins in innate host defense mechanisms against pathogens. J. Innate. Immun. 1, 123-135. doi: 10.1159/000163037

Doyle, T. B., Hawkins, A. C., and McCarter, L. L. (2004). The complex flagellar torque generator of Pseudomonas aeruginosa. J. Bacteriol. 186, 6341-6350. doi: 10.1128/JB.186.19.6341-6350.2004

Gellatly, S. L., and Hancock, R. E. (2013). Pseudomonas aeruginosa: new insights into pathogenesis and host defenses. Pathog. Dis. 67, 159-173. doi: 10.1111/2049-632X.12033

Hatano, K., Matsumoto, T., Furuya, N., Hirakata, Y., and Tateda, K. (1996). Role of motility in the endogenous Pseudomonas aeruginosa sepsis after burn. J. Infect. Chemother. 2, 240-246. doi: 10.1007/BF02355121

Hayashi, N., Matsukawa, M., Horinishi, Y., Nakai, K., Shoji, A., Yoneko, Y., et al. (2013). Interplay of flagellar motility and mucin degradation the bacterium's flagellar filament rotational speed, a factor that facilitates the penetration of $P$. aeruginosa through the mucin layer. Recently, Shieh et al. reported that human airway epithelial cells secrete GRO- $\alpha$ (Shieh et al., 2014). Therefore, GRO- $\alpha$ might induce the $P$. aeruginosa cystic fibrosis and pneumonia by mechanisms identical to those of our findings.

In summary, our study using in vitro intestinal epithelial cells showed that small proteins of $<10 \mathrm{kDa}$ secreted by these cells, including GRO- $\alpha$ and other as yet unidentified ones, may be important factors in the initiation of $P$. aeruginosa translocation. Further studies are required to clarify the relation between host cells and $P$. aeruginosa. Ultimately, understanding these mechanisms may lead to new therapeutic strategies for $P$. aeruginosa gut-derived sepsis.

\section{AUTHOR CONTRIBUTIONS}

$\mathrm{NH}$ and NG designed this research. NH, AY, MY, MK, MM, CF, and NN performed experiments. $\mathrm{NH}$ and $\mathrm{AY}$ analyzed the data. $\mathrm{NH}$ and AY drafted the manuscript. All authors have read the manuscript and approved its submission.

\section{FUNDING}

This work was supported by JSPS KAKENHI Grant Numbers 16K19129 and 26860295, and the Kyoto Pharmaceutical University Fund for the Promotion of Scientific Research to NH. This work was also funded by the MEXT Supported Program for the Strategic Research Foundation at Private Universities, Japan, 2013-2017, to NG.

\section{ACKNOWLEDGMENTS}

We are grateful to Dr. Masataka Oda for useful discussion and advice. The authors would like to thank Enago (www.enago.jp) for the English language review. stimulates the association of Pseudomonas aeruginosa with human epithelial colorectal adenocarcinoma (Caco-2) cells. J. Infect. Chemother. 19, 305-315. doi: 10.1007/s10156-013-0554-4

Hayashi, N., Nishizawa, H., Kitao, S., Deguchi, S., Nakamura, T., Fujimoto, A., et al. (2015). Pseudomonas aeruginosa injects type III effector ExoS into epithelial cells through the function of type IV pili. FEBS Lett. 589, 890-896. doi: 10.1016/j.febslet.2015.02.031

Heeb, S., Itoh, Y., Nishijyo, T., Schnider, U., Keel, C., Wade, J., et al. (2000). Small, stable shuttle vectors based on the minimal pVS1 replicon for use in gramnegative, plant-associated bacteria. Mol. Plant Microbe Interact. 13, 232-237. doi: 10.1094/MPMI.2000.13.2.232

Janeway, C. A., and Medzhitov, R. (2002). Innate immune recognition. Annu. Rev. Immunol. 20, 197-216. doi: 10.1146/annurev.immunol.20.083001. 084359

Kanda, Y. (2013). Investigation of the freely available easy-to-use software 'EZR' for medical statistics. Bone Marrow Transplant. 48, 452-458. doi: 10.1038/bmt.2012.244

Kato, J., Kim, H. E., Takiguchi, N., Kuroda, A., and Ohtake, H. (2008). Pseudomonas aeruginosa as a model microorganism for investigation of chemotactic behaviors in ecosystem. J. Biosci. Bioeng. 106, 1-7. doi: $10.1263 /$ jbb.106.1 
Kessler, E., Israel, M., Landshman, N., Chechick, A., and Blumberg, S. (1982). In vitro inhibition of Pseudomonas aeruginosa elastase by metal-chelating peptide derivatives. Infect. Immun. 38, 716-723.

Koh, A. Y., Mikkelsen, P. J., Smith, R. S., Coggshall, K. T., Kamei, A., Givskov, M., et al. (2010). Utility of in vivo transcription profiling for identifying Pseudomonas aeruginosa genes needed for gastrointestinal colonization and dissemination. PLoS ONE 5:e15131. doi: 10.1371/journal.pone.0015131

Liu, X., and Parales, R. E. (2008). Chemotaxis of Escherichia coli to pyrimidines: a new role for the signal transducer tap. J. Bacteriol. 190, 972-999. doi: 10.1128/JB.01590-07

Macnab, R. M. (2003). How bacteria assemble flagella. Annu. Rev. Microbiol. 57, 77-100. doi: 10.1146/annurev.micro.57.030502.090832

Markou, P., and Apidianakis, Y. (2014). Pathogenesis of intestinal Pseudomonas aeruginosa infection in patients with cancer. Front. Cell. Infect. Microbiol. 3:115. doi: 10.3389/fcimb.2013.00115

Miller, W. G., and Lindow, S. E. (1997). An improved GFP cloning cassette designed for prokaryotic transcriptional fusions. Gene 191, 149-153. doi: 10.1016/S0378-1119(97)00051-6

Murray, T. S., and Kazmierczak, B. I. (2008). Pseudomonas aeruginosa exhibits sliding motility in the absence of type IV pili and flagella. J. Bacteriol. 190, 2700-2708. doi: 10.1128/JB.01620-07

Ohara, T., and Itoh, K. (2003). Significance of Pseudomonas aeruginosa colonization of the gastrointestinal tract. Intern. Med. 42, 1072-1076. doi: 10.2169/internalmedicine.42.1072

Okuda, J., Hayashi, N., Arakawa, M., Minagawa, S., and Gotoh, N. (2013). Type IV pilus protein PilA of Pseudomonas aeruginosa modulates calcium signaling through binding the calcium-modulating cyclophilin ligand. J. Infect. Chemother. 19, 653-664. doi: 10.1007/s10156-012-0536-y

Okuda, J., Hayashi, N., Okamoto, M., Sawada, S., Minagawa, S., Yano, Y., et al. (2010). Translocation of Pseudomonas aeruginosa from the intestinal tract is mediated by the binding of ExoS to an Na,K-ATPase regulator, FXYD3. Infect. Immun. 78, 4511-4522. doi: 10.1128/IAI.00428-10

Qian, C., Wong, C. C., Swarup, S., and Chiam, K. H. (2013). Bacterial tethering analysis reveals a "run-reverse-turn" mechanism for Pseudomonas species motility. Appl. Environ. Microbiol. 79, 4734-4743. doi: 10.1128/AEM.01027-13

Rashid, M. H., and Kornberg, A. (2000). Inorganic polyphosphate is needed for swimming, swarming, and twitching motilities of Pseudomonas aeruginosa. Proc. Natl. Acad. Sci. U.S.A. 97, 4885-4890. doi: 10.1073/pnas.060030097

Sallenave, J. M. (2014). Phagocytic and signaling innate immune receptors: are they dysregulated in cystic fibrosis in the fight against Pseudomonas aeruginosa. Int. J. Biochem. Cell Biol. 52, 103-107. doi: 10.1016/j.biocel.2014.01.013

Sampedro, I., Parales, R. E., Krell, T., and Hill, J. E. (2015). Pseudomonas chemotaxis. FEMS. Microbiol. Rev. 39, 17-46. doi: 10.1111/1574-6976.12081

Shieh, J. M., Tsai, Y. J., Tsou, C. J., and Wu, W. B. (2014). CXCL1 regulation in human pulmonary epithelial cells by tumor necrosis factor. Cell. Physiol. Biochem. 34, 1373-1384. doi: 10.1159/000366344
Shikata, M., Hayashi, N., Fujimoto, A., Nakamura, T., Matsui, N., Ishiyama, A., et al. (2016). The pilT gene contributes to type III ExoS effector injection into epithelial cells in Pseudomonas aeruginosa. J. Infect. Chemother. 22, 216-220. doi: 10.1016/j.jiac.2015.12.012

Shimizu, K., Ogura, H., Goto, M., Asahara, T., Nomoto, K., Morotomi, M., et al. (2006). Altered gut flora and environment in patients with severe SIRS. J. Trauma 60, 126-133. doi: 10.1097/01.ta.0000197374.99755.fe

Shitashiro, M., Kato, J., Fukumura, T., Kuroda, A., Ikeda, T., Takiguchi, N., et al. (2003). Evaluation of bacterial aerotaxis for its potential use in detecting the toxicity of chemicals to microorganisms. J. Biotechnol. 101, 11-18. doi: 10.1016/S0168-1656(02)00285-7

Stover, C. K., Pham, X. Q., Erwin, A. L., Mizoguchi, S. D., Warrener, P., Hickey, M. J., et al. (2000). Complete genome sequence of Pseudomonas aeruginosa PAO1, an opportunistic pathogen. Nature 406, 959-964. doi: 10.1038/350 23079

Tlaskalova-Hogenova, H., Stepankova, R., Kozakova, H., Hudcovic, T., Vannucci, L., Tuckova, L., et al. (2011). The role of gut microbiota (commensal bacteria) and the mucosal barrier in the pathogenesis of inflammatory and autoimmune diseases and cancer: contribution of germ-free and gnotobiotic animal models of human diseases. Cell. Mol. Immunol. 8, 110-120. doi: 10.1038/cmi. 2010.67

Toutain, C. M., Zegans, M. E., and O’Toole, G. A. (2005). Evidence for two flagellar stators and their role in the motility of Pseudomonas aeruginosa. J. Bacteriol. 187, 771-777. doi: 10.1128/JB.187.2.771-777.2005

Tremblay, J., Richardson, A. P., Lepine, F., and Deziel, E. (2007). Selfproduced extracellular stimuli modulate the Pseudomonas aeruginosa swarming motility behaviour. Environ. Microbiol. 9, 2622-2630. doi: 10.1111/j.1462-2920.2007.01396.x

Vincent, J. L., Rello, J., Marshall, J., Silva, E., Anzueto, A., Martin, C. D., et al. (2009). International study of the prevalence and outcomes of infection in intensive care units. JAMA 302, 2323-2329. doi: 10.1001/jama.2009.1754

Wu, L., Estrada, O., Zaborina, O., Bains, M., Shen, L., Kohler, J. E., et al. (2005). Recognition of host immune activation by Pseudomonas aeruginosa. Science 309, 774-777. doi: 10.1126/science.1112422

Conflict of Interest Statement: The authors declare that the research was conducted in the absence of any commercial or financial relationships that could be construed as a potential conflict of interest.

Copyright (C) 2017 Hayashi, Yokotani, Yamamoto, Kososhi, Morita, Fukunishi, Nishizawa and Gotoh. This is an open-access article distributed under the terms of the Creative Commons Attribution License (CC BY). The use, distribution or reproduction in other forums is permitted, provided the original author(s) or licensor are credited and that the original publication in this journal is cited, in accordance with accepted academic practice. No use, distribution or reproduction is permitted which does not comply with these terms. 